\title{
Pachydermoperiostosis: An unusual cause of arthritis
}

\author{
Fariba Binesh ${ }^{1}$, Naser Ali Mirhosseini ${ }^{2}$, Ali Dehghan ${ }^{3}$, Pegah Kamal $^{1}$, Elahah Mansouri \\ Moghadam ${ }^{2}$
}

1 Department of Pathology, Shahid Sadoughi University of Medical Sciences, Yazd, Iran

2 Department of Pediatrics, Shahid Sadoughi University of Medical Sciences, Yazd, Iran

3 Department of Internal Medicine, Shahid Sadoughi University of Medical Sciences, Yazd, Iran

*Corresponding Author: Dr. Fariba Binesh, Department of Pathology, Shahid Sadoughi University of Medical Sciences, Yazd, Iran. Email: binesh44@yahoo.com

Received: November 02, 2019; Accepted: December 08, 2019

\begin{abstract}
Pachydermoperiostosis (PDP) is a curious genodermatosis, defined by pachydermia, digital clubbing, periostosis and touches mostly men. Its pathogenesis is unknown and diagnosis can be made on the grounds of classical clinical and imaging manifestations. Here we present a 22 yearold man introduced with skeletal marks, pachyderma and clubbing of fingers and toes,which begun at the age of 6 months. X-rays revealed mild hyperperiostosis. Mention should be made that clinical signs of PDP can be misinterpreted with secondary hypertrophic osteoarthropathy, thyroid acropachy, acromegaly and some rheumatologic disorders. There is no definite remedy for its symptoms. The patients need a precise follow-up due to problems that may happen in the long-range.
\end{abstract}

Keywords: Pachyderma, Clubbing, Periostosis, Hypertrophic Osteoarthropathy.

\section{INTRODUCTION}

Hypertrophic osteoarthropathy (HOA) can be divided into primary or secondary forms. Almost five percent of HOA are primary although its precise prevalence in the general public is obscure [1]. A prevalence of $0.16 \%$ has been presented by Jajic and Jajic [2]. PDP or primary hypertrophic osteoarthropathy, is an autosomal-dominant/autosomal recessive disease with inconstant presentation. Secondary HOAs stand with underlying disorders such as malignancy [3] and cardiopulmonary disorders [4]. PDP can be distinguished by the existence of the triad of digital clubbing, periostosis, and pachydermia [5]. Signs usually become visible at about puberty. Three types have been explained: a complete form with pachyderma, digital clubbing and periostitis, an incomplete form with skeletal changes but without pachyderma, and a forme frusta with eminent pachyderma and the least bony abnormalities. An infantile type has also been characterized [6]. Here in, we present a 22 year-old man with chief complaints of ankle pain, clubbing of fingers and toes along with plenty of sweating and progressive roughen of facial appearences. Physical examination, laboratory findings, and imagings all confirmed a diagnosis of PDP.

\section{CASE REPORT}

A 22-year-old Muslim male borne out of a consanguineous marriage referred to our outpatient clinic with chief complaints of pain and swelling in his ankles and knees which he had for several years. The ache was mischievous in the beginning, pulsates in character and not soothed by analgesics. He also complained of plenty of sweating, progressive bigness of fingers and toes and imperceptible roughing of facial aspects. According to his mother, inception was gentle from the age of 6 months. Physical examination of the patient showed plenty sweating, seborrhea, acne (fig-1), horizontal and vertical furrowing on the forehead (fig-2) and a prominent nose (fig- 3). The patient had concurrent clubbing of fingers and toes and ankle and knee joint effusion (fig-4,5). He also had double rows of teeth (fig-6). Cardiovascular, respiratory, neurological, and thyroid evaluation was done and that was normal. Thyroid function test, rheumatoid factor, and anti-cyclic citrullinated peptide were unremarkable. Other laboratory tests such as hematology, biochemistry and serology tests were normal. Radiographs of his leg revealed a cortical thickening in tibia and fibula (fig-7). The patient has a younger sister who is about 8 years of age and she also has clubbing of fingers and toes (fig$8,9)$. According to the above descriptions the diagnosis was familial HAO. Treatment was started with colchicines and NSAID. Regrettably, the financial situation of the patient hampered implementation of genetic studies. 


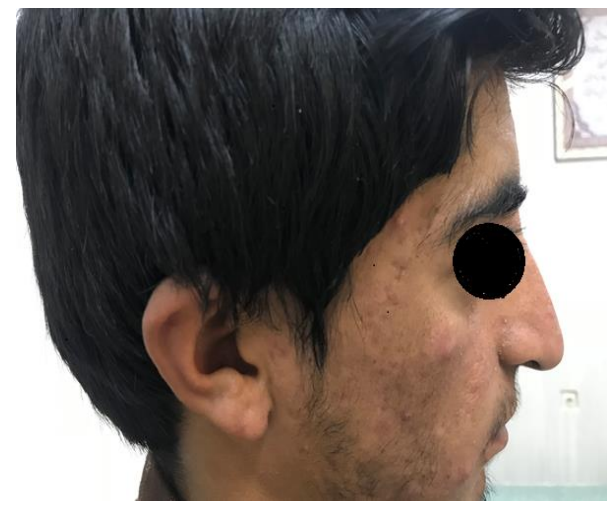

Fig 1

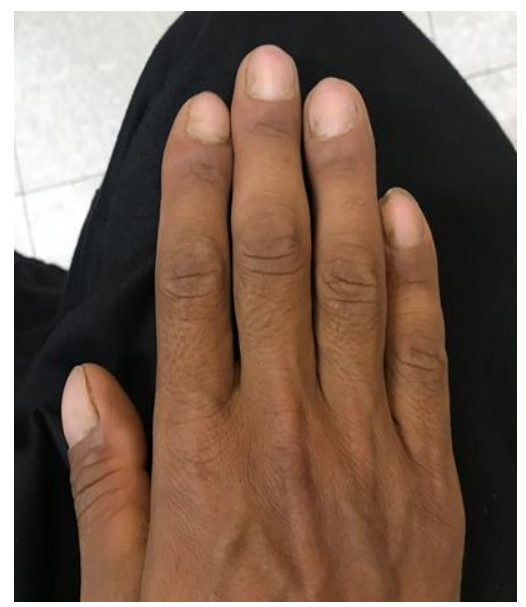

Fig 4

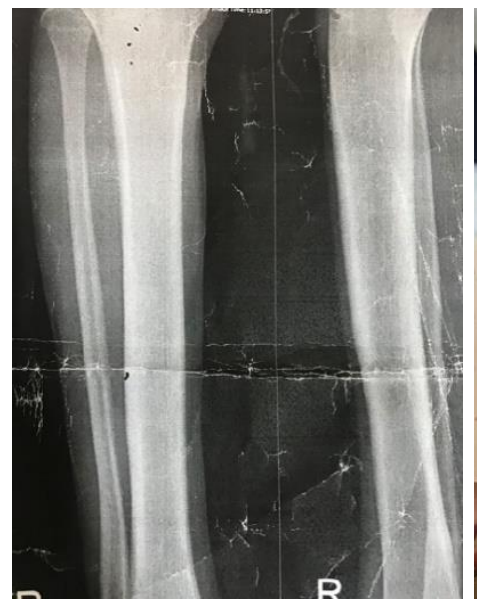

Fig 7

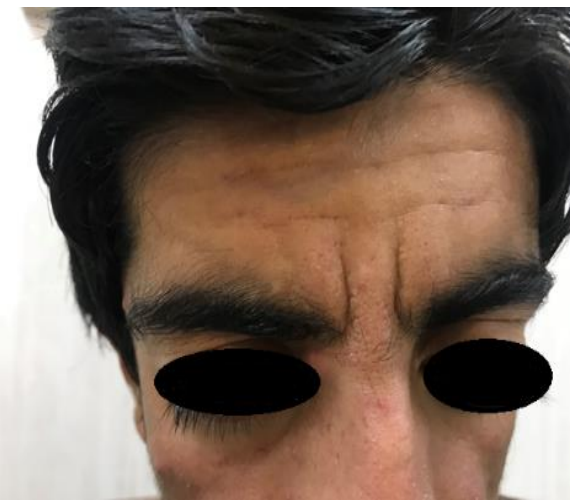

Fig 2

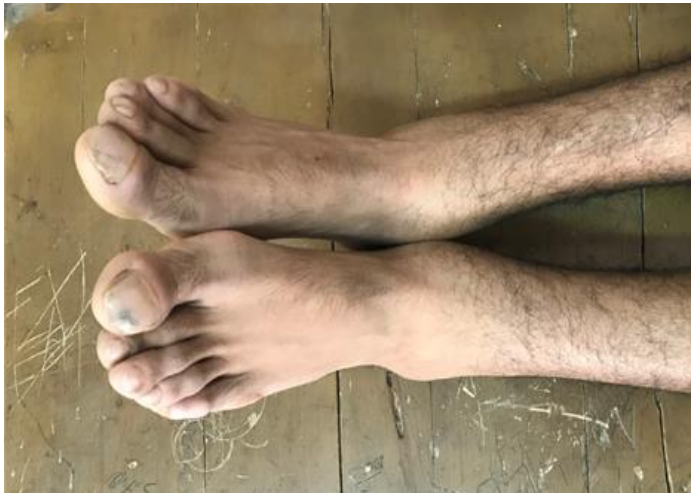

Fig 5

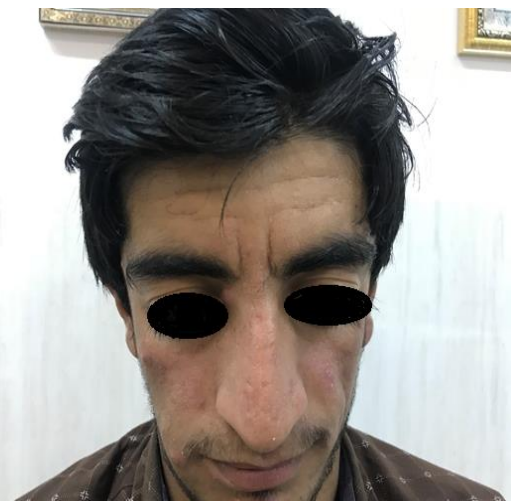

Fig 3

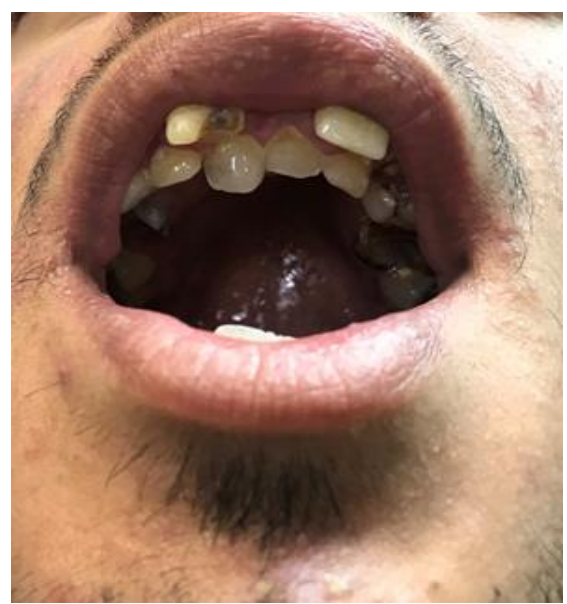

Fig 6

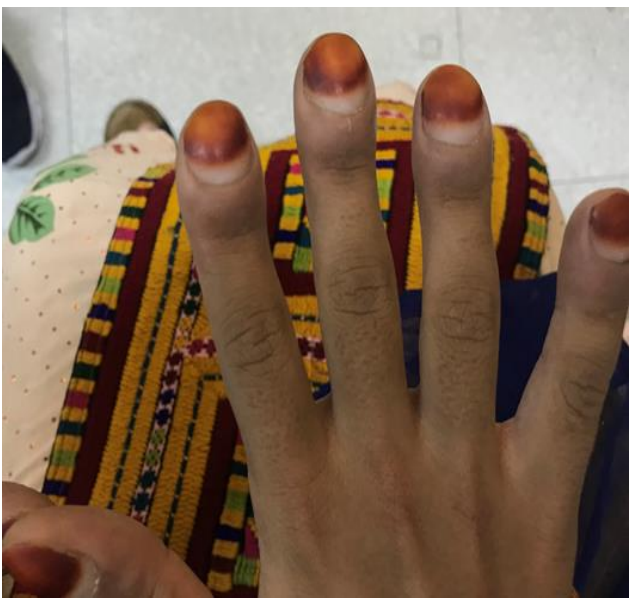

Fig 8

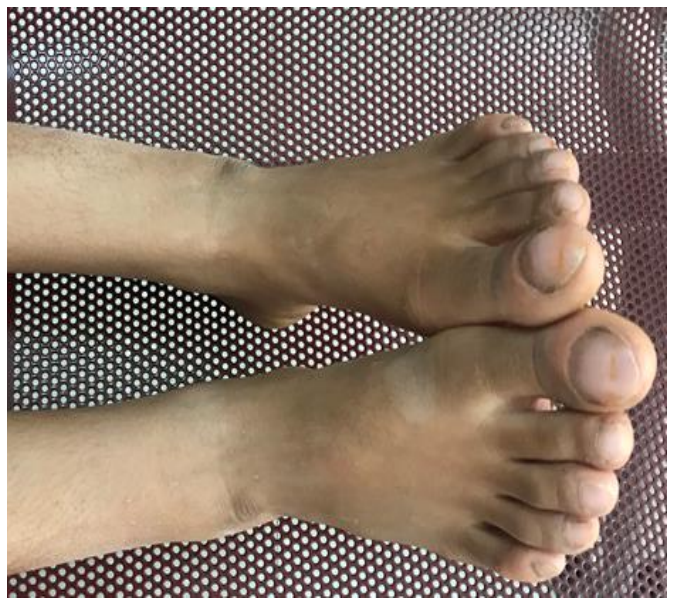

Fig 9

\section{DISCUSSION}

$\mathrm{HOA}$ is a syndrome determined by the triad of periostitis, digital clubbing, and pachydermia [7]. The major criteria comprise of periostosis, pachyderma and digital clubbing [8]. The minor criteria includes blepharoptosis, hyperhidrosis, arthralgia, seborrhea, joint effusion,gastric ulcer, cutis verticis gyrate, flushing oedema, acne and column like legs. The present case had three of the major criteria (digital clubbing, bony changes on skeletal radiograph and skin thickening) and six of the minor criteria (joint effusion, sweating, seborrhea, acne. arthralgia and column like legs), therefore was adequate to tag him as a case of complete pachydermoperiostosis. Precise inducement is obscure but newly mutations in $15-\mathrm{OH}$ prostaglandin dehydrogenase on chromosome $4 \mathrm{q} 34.1$ have been proposed as the conceivable etiology. A genetic study was designed for our patient, but could not be done due to financial limitation of the case. Symptoms usually start in teenage years but in our patient the signs began in the infancy period [9]. The clinical and radiological features in these patients can be misinterpreted with other disorders such as syphilitic periostitis, psoriatic onychopachydermoperiostitis (POPP), Paget's disease, and 
especially acromegaly [10]. Secondary HOA is accompanied with latent cardiopulmonary disorders, malignant diseases, thyroid acropachy, acromegaly, and chronic inflammatory rheumatic diseases. So, a careful investigation for the latent disease should be performed. It is told that involvement of the epiphyseal region distinguished PDP from the secondary one, in which the epiphyses are usually waived [8]. A spectrum of benign and malignant disordres [11] have been reported to accompany with PDP. These comprise squamous cell carcinoma, hypertrophic gastritis, peptic ulcer, gastric adenocarcinoma, Crohn's disease and myelofibrosis. Though arthritis has been presented in these patients is noninflammatory in character, Binit Vaidya, et al. reported two patients of PDP with inflammatory arthritis [12]. In addition, Hei Sung Kim et al. presented a case of PDP in a patient with hyperIgE syndrome [13]. No particular therapy is currently accessible. Medical handling [14] is recommended for symptomatic profit and it contains non-steroidal anti-inflammatory drugs, colchicine, corticosteroids and retinoids. Botulinum toxin- $A$ has also been used for cosmetic causes. Surgical approach comprises repair of skeletal malformation and plastic operation for deformities. Prospect for these patients is good and patients can have on ordinary lifetime. Our patient was counseled to be on an orderly follow-up and has currently only received NSAID and colchicine.

\section{CONCLUSION}

Although PDP is a benign disease, the disease makes a communal mark and directs towards a significant decrease in the patient's modality of life. Therefore, these patients should be managed by a group that includes dermatologists, rheumatologists and psychiatrists. PDP should be contemplated as differential diagnosis when a patient referrer with clubbing and acromegaloid manifestations. Specific physical manifestations with characteristic imaging findings assist in confirming correct diagnosis. It is substantial to diagnose this state because a misinterpretation may lead the case to undue examinations.

\section{Acknowledgement}

The authors wish to thank the Management and Staff of the Federal Medical Center, Owerri, Imo State, Nigeria particularly the outpatient staff for their support during the study. All the patients who participated in this study are also acknowledged.

\section{Conflict of Interest}

We declare that we have no conflict of interest.

\section{Financial Support}

None declared.

\section{REFERENCES}

1. Touraine A, Solente G, Golẻ L. Un syndrome osteodermatopathique: La pachydermieplicaturẻẻ avec pachyẻriostose des extrẻmitẻs.Press Med 1935;43:1820-4.

2. Jajic Z, Jajic I. Prevalence of primary hypertrophic osteoarthropathy in selected population. Clin Exp Rheum 1992;10:73.

3. Rimoin DL. Pachydermoperiostosis (idiopathic clubbing and periostosis): Genetic and physiologic considerations. N Engl J Med 1965;272:923-31.

4. Amir Joshi, Gaurav Nepal,Yow Ka Shing, Hari Prasad Panthi and et al. Pachydermoperiostosis (Touraine-Solente-Gole syndrome): a case report Journal of Medical Case Reportsvolume 13, Article number: 39 (2019).

5. Castori M, Sinibaldi L, Mingarelli R, Lachman RS, Rimoin DL, Dallapiccola B. Pachydermoperiostosis: an update. Clin Genet 2005; 68: 477-486.

6. Reginato AJ, Schiapachasse V, Guerrero R. Familial idiopathic hypertrophic osteoarthropathy and cranial suture defects in children. Skeletal Radiol 1982;8:105-9.

7. Kowalewski M, Urban M, Górska A. Familial occurrence of primary hypertrophic osteoarthropathy: A case report. Med Sci Monit 1997;3:229-34.

8. Rastogi R, Suma GN, Prakash R, et al. Pachydermoperiostosis or primary hypertrophic osteoarthropathy: A rare clinicoradiologic case. Indian J Radiol Imaging 2009;19:123-6.

9. Matucci-Cerinic M, Lotti T, Jajic I, Pignone A, Bussani C, Cagnoni M. The clinical spectrum of pachydermoperiostosis (primary hypertrophic osteoarthropathy). Medicine 1991;70:208-14.

10. Uppal S, Diggle CP, Carr IM, Fishwick CW, Ahmed M, Ibrahim $\mathrm{GH}$, et al. Mutations in 15-hydroxyprostaglandin dehydrogenase cause primary hypertrophic osteoarthropathy. Nature Genet 2008;40:789-93.

11. Bachmeyer C, Blum L, Cadranel JF, et al. Myelofibrosis in a patient with pachydermoperiostosis. Clin Exp Dermatol 2005;30:646-8.

12. Binit Vaidya, Rikesh Baral, Hritu Baral and Shweta Nakarmi, Inflammatory variant of pachydermoperiostosis responding to methotrexate: a report of two cases, Oxford Medical Case Reports, 2019;4, 160-164.

13. Kim HS, Teo RY, Tan AW. Cutis verticis gyrata in a patient with hyper-lgE syndrome. Acta dermato-venereologica. 2009;89(4):413-4.

14. Kabi F, Mkinsi O, Janani S, et al. A case report. J Intern Med 2006;27:710-2. 